\title{
A Fully Data-Driven Approach for Leak Localization in Water Distribution Networks*
}

\author{
Luis Romero $^{1 \star}$, Vicenç Puig ${ }^{1}$, Gabriela Cembrano ${ }^{1,2}$, Joaquim Blesa ${ }^{1}$ and Jordi Meseguer ${ }^{2}$
}

\begin{abstract}
This paper presents a data-driven technique for the localization of leaks in water distribution networks (WDN). The methodology requires hydraulic data, i.e., pressure measurements from a set of sensors installed throughout the network and topological information. Therefore, the hydraulic model of the WDN is not necessary for its operation. The hydraulic state of the complete set of nodes of the network is approximated by means of a graph-based interpolation technique. Then, a set of candidates where the leak can be located is achieved by comparing the computed states for both the leaky and nominal cases. The methodology is applied to a case study based on a real network, providing and discussing several graphical results and key performance indicators.
\end{abstract}

\section{INTRODUCTION}

The detection and localization of leaks in water distribution networks (WDNs) is of great significance for water utilities. Water losses may account for up to a $30 \%$ of the total amount of distributed water [1], and hence the operational costs are excessively high. Considering this along with the worldwide growing demand of water (increasing by $55 \%$ between 2000 and 2050 according to [2]) and the associated environmental [3] and health [4] problems, the great interest in the research of this field is evident (see [5] for an extensive review).

Regarding the leak localization problem, the majority of the most extended techniques are classified as model-based, i.e., they rely on a hydraulic model of the WDN. A wellknown approach is proposed in [6], based on the comparison of pressure residuals against a fault signature matrix obtained by means of hydraulic simulations. The sensitivity analysis is also considered in [7], along with a search-space reduction approach.

However, while model-based approaches lead to good results under ideal conditions, the presence of model errors like nodal demand uncertainty and measurements noise can

\footnotetext{
*The authors want to thank the RIS3CAT Utilities 4.0 SENIX project (COMRDI16-1-0055), as well as the Spanish national project DEOCS (DPI2016-76493-C3-3-R) and the Spanish State Research Agency through the María de Maeztu Seal of Excellence to IRI (MDM-2016-0656). Joaquim Blesa is a Serra Húnter Fellow.

${ }^{1}$ L. Romero (luis.romero.ben@upc.edu; corresponding author ${ }^{\star}$ ), V. Puig (vicenc.puig@upc.edu), G. Cembrano (gabriela.cembrano@upc.edu) and J. Blesa (joaquim.blesa@upc.edu) are with Institut de Robòtica i Informàtica Industrial (CSIC-UPC). Carrer Llorens Artigas, 4-6, 08028, Barcelona, Spain; and with the Supervision, Safety and Automatic Control Research Center (CS2AC) of the Universitat Politécnica de Catalunya, Campus de Terrassa, Gaia Building, Rambla Sant Nebridi, 22, 08222 Terrassa, Barcelona, Spain

${ }^{2}$ J. Meseguer (jmeseguer@cetaqua.com) and G. Cembrano are with CETaqua, Water Technology Centre (SUEZ Spain Group), Ctra. d'Esplugues, 75, 08940, Cornellà del LLobregat, Barcelona, Spain
}

deteriorate the performance [8]. These problems can be diminished by data-driven approaches [9] due to their reduced dependency on a model of the WDN.

This paper presents a data-driven method based on two complementary stages (a detection algorithm must be exploited to monitor the appearance of leaks and notify their existence so that the localization approach can be used):

- A graph-based interpolation of the hydraulic state of the network that utilizes the water system available information, i.e., measurements from installed pressure sensors. The recovery of the complete WDN state from a set of values is a common stage to several data-driven strategies. In [10], a two-phase approach employing network decomposition and Gauss-Newton Belief Propagation (GN-BP) inference is proposed to obtain quasi-optimal states.

- A comparison between leaky and healthy states, selecting as a result a set of nodes as possible origins of the leak. Again, similar comparisons are exploited throughout the literature. For example in [11], which also exploits interpolation techniques, the pressure maps achieved for leak and leak-free operations are compared.

\section{METHODOLOGY}

The basic procedure of the proposed leak localization methodology is summarised in Fig. 1. This strategy uses two different types of information.

On the one hand, hydraulic measurements are gathered by a set of pressure sensors distributed throughout the nodes of the WDN. The data is processed in order to operate with hydraulic heads, i.e., measured pressure added to node elevation. These sensors not only provide information of current measurements at a leak event but also allow to collect historical data from leak-free scenarios.

On the other hand, structural information of the network is required (concretely, the length of the pipes connecting the different nodes).

Both sources of information are exploited by a graphbased interpolation procedure to approximate the complete state of the network from the hydraulic measurements, i.e., to estimate the unknown hydraulic values at the nodes without sensor (see [12] for further explanations about the usage of interpolation approaches for leak localization). This operation is performed over the data with and without leaks in order to reconstruct the complete state for both scenarios. These data vectors are compared from a geometric perspective, so that the set of leak candidates is selected by means of a computed metric. 


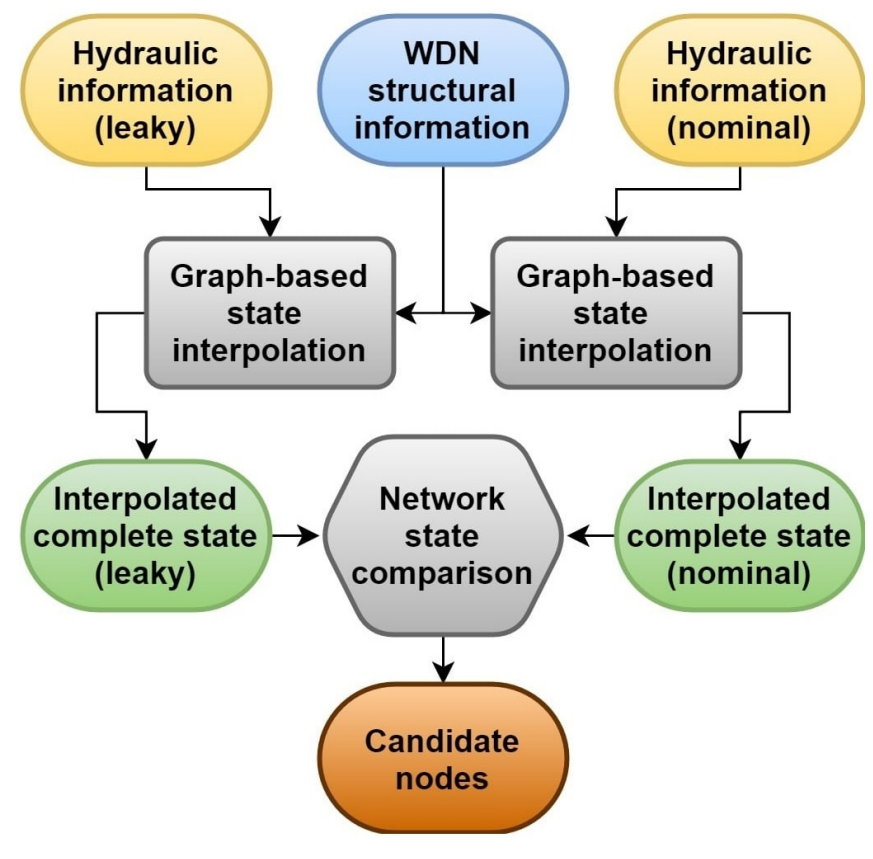

Fig. 1. Schematic diagram of the methodology operation.

Due to the design of the presented strategy, the scheme in Fig. 1 operates using the measurements from a single time instant. Nevertheless, time integration can be naturally implemented: the methodology is embedded into a process that uses the hydraulic information of each time instant to compute the previously mentioned metric and sums cumulatively the result over a selected time window. Then, the candidates are obtained from this cumulative sum.

\section{A. Graph-based state interpolation}

Let us consider a simple directed graph $\mathscr{G}=(\mathscr{V}, \mathscr{E})$ representing the structure of the studied WDN. $\mathscr{V}$ stands for the set of nodes of the graph, i.e., junctions of the network; and $\mathscr{E}$ represents the set of edges of the graph, i.e., pipes of the network. The number of nodes in the graph is represented by the node set cardinality as $|\mathscr{V}|$, and analogously the number of pipes is $|\mathscr{E}|$. A node is denoted as $v_{i} \in \mathscr{V}$, and an edge $e_{i j}=\left(v_{i}, v_{j}\right) \in \mathscr{E}$ has $v_{i}$ as its source and $v_{j}$ as its sink. A pair of nodes in $\mathscr{V}$ will be said to be adjacent if they are connected, independently of the associated directionality.

Besides, the relation between nodes $v_{i}$ and $v_{j}$ will be represented by the $i-j$ component of the weighted adjacency matrix $W \in \mathbb{R}^{|\mathscr{V}| \times|\mathscr{V}|}$ associated to graph $\mathscr{G}$. In this work, this matrix is composed as follows:

$$
w_{i j}= \begin{cases}\frac{1}{c_{i j}}, & \text { if }\left(e_{i j} \in \mathscr{E} \text { or } e_{j i} \in \mathscr{E}\right) \text { and } c_{i j} \neq 0 \\ 0, & \text { otherwise }\end{cases}
$$

where $c_{i j}$ (equal to $c_{j i}$ ) denotes the length of the pipe connecting the junctions represented by nodes $v_{i}$ and $v_{j}$.

Consider a function $f: \mathscr{V} \rightarrow \mathbb{R}$ on the graph $\mathscr{G}$ which assigns a real value $f(i)$ to each node $v_{i}$. Henceforth, each value $f(i)$ of $f$ will be denoted as $x_{i}$ and will be considered as the state in node $i$, as the function $f$ can be represented as a column vector in $\mathbb{R}^{|\mathscr{V}|}$, denoted from now on as $\mathbf{x}$.

The main objective of the graph-based interpolation procedure is the propagation of the information about the measured node states (hydraulic heads) to achieve estimations of the unknown ones. To do so, a relaxation on the non-linear relation among the hydraulic heads of adjacent junctions is applied: the state (i.e, approximation of its hydraulic head) of a certain node is considered to be a weighted linear contribution of the states of its neighbours:

$$
x_{i}=\frac{1}{d_{i}} \sum_{j=1}^{|\mathcal{N}(i)|} w_{i j} x_{j}=\frac{1}{d_{i}} \mathbf{w}_{i} \mathbf{x}
$$

where $\mathbf{w}_{i}$ denotes the $i$-th row of the weighted adjacency matrix $W, \mathscr{N}(i) \in \mathscr{V}$ represents the set of adjacent nodes to $v_{i}$ and $d_{i}$ is known as the degree of node $v_{i}$, and it is computed as $d_{i}=\sum_{j=1}^{|\mathscr{N}(i)|} w_{i j}$. Considering $d_{i} \forall i=$ $1, \ldots,|\mathscr{V}|$, a diagonal degree matrix $D \in \mathbb{R}^{|\mathscr{V}| \times|\mathscr{V}|}$ can be defined as $D=\operatorname{diag}\left(d_{1}, d_{2}, \ldots, d_{i}, \ldots, d_{|\mathscr{V}|}\right)$.

The design of the $W$ is hence justified by the aim of the interpolation process: closer neighbouring nodes must contribute more to the state value of the considered one.

In order to impose the approximation proposed by (2), the state vector $\mathbf{x}$ should be computed to minimize the next expression:

$$
\sum_{i=1}^{|\mathscr{V}|}\left(x_{i}-\frac{1}{d_{i}} \mathbf{w}_{i} \mathbf{x}\right)^{2}=\left(\mathbf{x}-D^{-1} W \mathbf{x}\right)^{T}\left(\mathbf{x}-D^{-1} W \mathbf{x}\right)
$$

The available hydraulic information, i.e., the hydraulic heads at the sensorized nodes; is provided to the interpolation process by means of an equality constraint restricting the possible solutions:

$$
S \mathbf{x}=\mathbf{x}^{s}
$$

where $S \in \mathbb{R}^{|\mathscr{V}| \times|\mathscr{V}|}$ is a diagonal matrix whose $i$-th diagonal component indicates the presence a sensor at node $i$ by means of a value of 1 , whereas a value of 0 indicates that a sensor is not installed. The vector $\mathbf{x}^{s} \in \mathbb{R}^{|\mathscr{V}|}$ contains the head values of the sensorized nodes at the corresponding components, whereas the rest of elements are zero-valued.

In order to solve the presented problem from a quadratic programming perspective, the expression at (3) can be manipulated as follows:

$$
\begin{array}{r}
\left(\mathbf{x}-D^{-1} W \mathbf{x}\right)^{T}\left(\mathbf{x}-D^{-1} W \mathbf{x}\right)= \\
\left(\left(I_{|\mathscr{V}|}-D^{-1} W\right) \mathbf{x}\right)^{T}\left(\left(I_{|\mathscr{V}|}-D^{-1} W\right) \mathbf{x}\right)= \\
\left(D^{-1}(D-W) \mathbf{x}\right)^{T}\left(D^{-1}(D-W) \mathbf{x}\right)
\end{array}
$$

where $I_{|\mathscr{V}|}$ represents the identity matrix of size $|\mathscr{V}|$.

Considering $L \in \mathbb{R}^{|\mathscr{V}| \times|\mathscr{V}|}$ to be the unnormalized Laplacian of graph $\mathscr{G}$, computed as $L=D-W$ (it is a symmetric matrix, i.e., $L=L^{T}$ ), the final expression at (5) can be rearranged in the following way: 


$$
\begin{array}{r}
\left(D^{-1} L \mathbf{x}\right)^{T}\left(D^{-1} L \mathbf{x}\right)=\mathbf{x}^{T}\left(D^{-1} L\right)^{T}\left(D^{-1} L\right) \mathbf{x}= \\
\mathbf{x}^{T} L D^{-2} L \mathbf{x}
\end{array}
$$

Furthermore, a second goal is pursued during the operation of the graph-based interpolation process. If the directionality of the flow at the pipes of a WDN are imported into a directed graph, it would be acyclic (concretely, a directed acyclic graph or DAG), because water can not follow a cyclic path through the water system. Thus, it would be desirable to consider this feature while searching for a solution to the interpolation problem.

The directionality of graphs is represented by the oriented incidence matrix $B \in \mathbb{R}^{|\mathscr{E}| \times|\mathscr{V}|}$, which can be constructed as follows:

$$
b_{k j}=\left\{\begin{aligned}
1, & \text { if } e_{k}=\left(v_{i}, v_{j}\right) \in \mathscr{E} \\
-1, & \text { if } e_{k}=\left(v_{j}, v_{i}\right) \in \mathscr{E} \\
0, & \text { otherwise }
\end{aligned}\right.
$$

i.e., the chosen sign convention gives a value of -1 to the component of matrix $B$ considering edge $k$ and node $j$ if there exist an edge $e_{k}$ in the edge set $\mathscr{E}$ that has node $j$ as its source; and a value of 1 if there exist an edge $e_{k}$ that contains node $j$ as its sink. The value would be 0 for every other possible case.

In order to consider the graph directionality at the computation of the node state vector $\mathbf{x}$, the following constraint must be included:

$$
B \mathbf{x} \leq \gamma
$$

where $\gamma \in \mathbb{R}^{|\mathscr{V}|}$ is a vector whose components have a value of $\gamma \in \mathbb{R}$. Thus, this constraint imposes that the difference of states of the source and sink of an edge must be lower or equal to a threshold value $\gamma$, considering the sign convention in the design of $B$ : due to the node states approximating hydraulic heads, the desired flow directionality would be properly replicated if source nodes have a state value higher than sink nodes, as determined by the HazenWilliams equation, which relates the flow through a pipe with the characteristics of the pipe and the difference of pressure between its endpoints.

Ideally, the second term at (8) would be zero. However, the possible imprecisions in the available incidence matrix (it is commonly difficult or impossible to compute matrix $B$ exactly) force the inclusion of some flexibility in the constraint by means of the threshold $\gamma>0$. This parameter is included as a second term in the minimization problem to reach the smallest value that produces a feasible solution (considering the parallel attainment of the other objective).

Finally, the complete optimization problem can be arranged as follows:

$$
\begin{aligned}
\min _{\mathbf{x}, \gamma} & \frac{1}{2}\left[\mathbf{x}^{T} L D^{-2} L \mathbf{x}+\tau \gamma^{2}\right] \\
\text { s.t. } & B \mathbf{x} \leq \gamma \\
& \gamma>0 \\
& S \mathbf{x}=\mathbf{x}^{s}
\end{aligned}
$$

where the parameter $\tau$ measures the relative importance given to the second objective.

\section{B. Network state comparison}

Let us consider that a leak event has occurred and that the associated state vector $\mathbf{x}_{\text {leak }}$ is obtained by means of the graph-based interpolation procedure. In the same way, let us consider the availability of hydraulic information from a leak-free situation with similar boundary conditions. Its corresponding state vector $\mathbf{x}_{n o m}$ is computed through the interpolation process too.

Both state vectors can compose a matrix $X$ of the form $X=\left[\begin{array}{ll}\mathbf{x}_{\text {nom }} & \mathbf{x}_{\text {leak }}\end{array}\right] \in \mathbb{R}^{|\mathscr{V}| \times 2}$. Each row of the proposed matrix will correspond to a point in $\mathbb{R}^{2}$, where the $\mathrm{x}$-axis role is played by the nominal state values and the $y$-axis will represent the leaky state values. Thus, matrix $X$ can be regarded to contain a set of $|\mathscr{V}|$ two-dimensional points.

If two healthy scenarios are compared, the resulting points should be practically aligned in $\mathbb{R}^{2}$ (despite the line may not have unitary slope due to discrepancies in their boundary conditions).

However, the presence of a leak in one of the scenarios produces a difference between the computed and expected positions of some points, due to the effects of the leak in the hydraulic heads of the affected nodes. Concretely, the leaky node and the ones close to it undergo a reduction in their state value, and their associated points of $X$ are located below the best-fitting line of the complete cloud of points (as the majority of nodes would not suffer this state value reduction, they continue to be sufficiently aligned).

The methodology computes the least-squares fitting line for the cloud of points defined by $X$, and then calculates the distance from each point to the achieved line.

To perform the first operation, a first-order polynomial is generated to fit the cloud of points defined by matrix $X$ :

$$
\mathbf{x}_{\text {leak }}=X_{\text {nom }} \boldsymbol{\theta}
$$

where $X_{\text {nom }}=\left[\begin{array}{ll}\mathbf{x}_{\text {nom }} & \mathbf{1}_{|\mathscr{V}|}\end{array}\right] \in \mathbb{R}^{|\mathscr{V}| \times 2}$, with $\mathbf{1}_{|\mathscr{V}|}$ being a vector of length $|\mathscr{V}|$ whose components are all 1 ; and $\theta=$ $\left[\begin{array}{ll}\theta_{1} & \theta_{2}\end{array}\right]^{T} \in \mathbb{R}^{2}$. The value of $\boldsymbol{\theta}$ is computed by solving the least-squares problem, i.e.:

$$
\boldsymbol{\theta}=\left(X_{\text {nom }}^{T} X_{\text {nom }}\right)^{-1} X_{\text {nom }}^{T} \mathbf{x}_{\text {leak }}
$$

The signed perpendicular distance from an arbitrary point $\left(x_{p}, y_{p}\right)$ to a line $\ell(a, b, c)$ of the form $a x+b y+c=0$ is computed as:

$$
r\left(\left(x_{p}, y_{p}\right), \ell\right)=\frac{a x_{p}+b y_{p}+c}{\sqrt{a^{2}+b^{2}}}
$$


The sign information is kept due to the interest on points below the computed line.

Therefore, considering that our notation implies that $a=$ $\theta_{1}, b=-1$ and $c=\theta_{2}$, the calculation of a vector $\mathbf{r} \in$ $\mathbb{R}^{|\mathscr{V}|}$ that contains the signed perpendicular distance from each point in $X$ to their associated best-fitting line, can be performed by means of the following expression:

$$
\mathbf{r}=X_{\text {ext }} \boldsymbol{\theta}_{\text {ext }}
$$

where $X_{\text {ext }}=\left[\begin{array}{ll}X & \left.\mathbf{1}_{|\mathscr{V}|}\right]\end{array}\right] \in \mathbb{R}^{|\mathscr{V}| \times 3}$ and $\theta_{\text {ext }} \in \mathbb{R}^{3}$ is:

$$
\boldsymbol{\theta}_{\text {ext }}=\left[\frac{\theta_{1}}{\sqrt{\theta_{1}^{2}+1}} \frac{-1}{\sqrt{\theta_{1}^{2}+1}} \frac{\theta_{2}}{\sqrt{\theta_{1}^{2}+1}}\right]^{T}
$$

Finally, due to the large set of nodes that may fulfil the requisite of having an associated point in $\mathbb{R}^{2}$ below the bestfitting line, that is, having a positive distance value; they need to be filtered. This problem is overcome by computing the standard deviation of $\mathbf{r}$ and employing the achieved value as a threshold that the distance $r_{i}$ of an arbitrary node $v_{i}$ must surpass to consider that node as a candidate to be the origin of the leak.

Moreover, the values at vector $\mathbf{r}$ allow to order the set of candidates, so that nodes with a higher distance value should be considered as the leak origin before nodes with a lower value.

In comparison with methodologies based on residuals computation (difference between the state of a node in leak and leak-free scenarios), the proposed approach computes a new metric that not only encodes information about the change of state for each individual node, but also considers the relation among the states of all the nodes of the network. This effect is produced because the computation of the bestfitting line directly depends on the $\mathbb{R}^{2}$ location of all the points in $X$, and hence, the relation between healthy and leaky states.

\section{CASE STUDY ANALYSIS}

A case study based on a real network is considered to apply the presented methodology and evaluate its performance. A schematic representation of the WDN is depicted in Fig. 2. The blue points represent the inner nodes of the WDN where sensors are not installed, whereas the green dots indicate the existence of a pressure meter. Finally, the magenta squares denote the location of the reservoirs, which are the water inlets to the network. A summary of the WDN most relevant properties is presented in Table I.

TABLE I

CASE STUDY NETWORK CHARACTERISTICS

\begin{tabular}{|c||c|}
\hline Feature & Value \\
\hline $\mathrm{N}^{0}$ of nodes & 4991 \\
$\mathrm{~N}^{0}$ of pressure sensors & 6 \\
$\mathrm{~N}^{\mathrm{o}}$ of reservoirs & 2 \\
$\mathrm{~N}^{\mathrm{o}}$ of pipes & 5153 \\
Total pipe length $(\mathrm{km})$ & 80 \\
Total area $\left(\mathrm{km}^{2}\right)$ & 6 \\
\hline
\end{tabular}

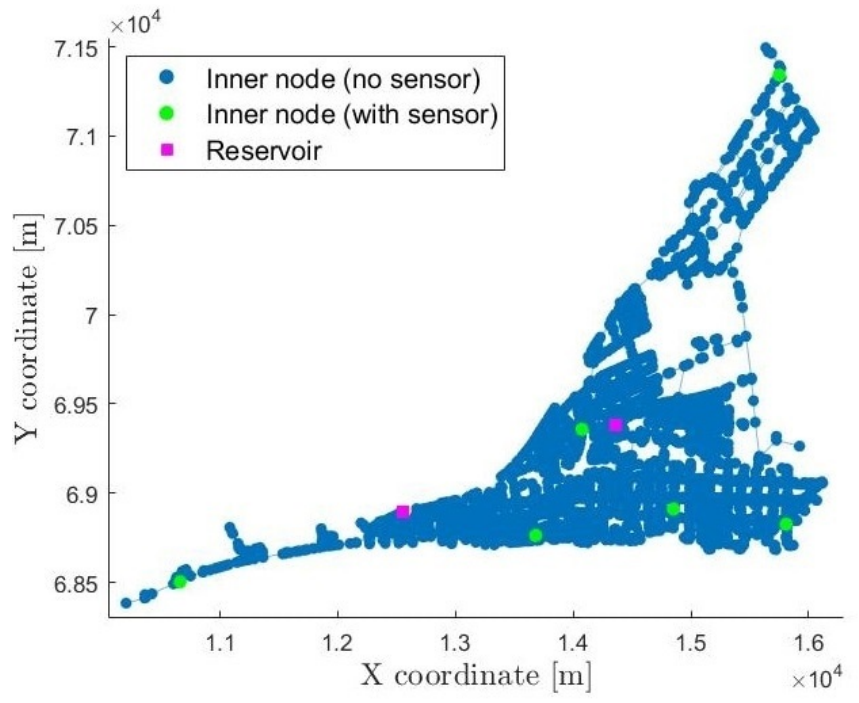

Fig. 2. Graph associated to the case study network.

The assessment of the methodology requires the availability of hydraulic information from leak and leak-free scenarios. They are simulated by means of a well-calibrated EPANET [13] model, replicating the conditions of the real network:

- Several sources of uncertainty are considered, affecting parameters like the nodal demands and pipe diameters and roughness.

- The sensors precision is limited to $\pm 0.1 \mathrm{~m}$ to match the properties of commonly used pressure meters.

- Different aspects like demand patterns, reservoirs outflow and valves operation are implemented to emulate the behaviour of a real scenario, as this information was available from the functioning of the real network.

Nevertheless, the sensorization density (conceived as the percentage of sensorized nodes) is almost negligible (a $0.12 \%$ ) if only considering the pressure sensors that are installed in the real network. The data-driven nature of the proposed leak localization method implies that these sensors are the only available source of hydraulic information about the network state. Thus, the addition of extra sensors must be carried out to properly assess the methodology. These extra metering devices are located by means of a sensor placement approach that seeks the reduction of the maximum (and mean) distance from a node to a sensor. The results for different leak events across the WDN are presented in Fig. 3 , considering the availability of 30 sensors (the 6 original sensors are maintained), that is a sensorization density of $0.6 \%$ with respect to the total nodes of the network.

These figures are formed by two differenced elements. On the one hand, a global representation of the WDN is included. The inner nodes with and without pressure meters are denoted with the same markers employed in Fig. 2. Moreover, the set of leak candidates is indicated by means of red dots, whereas the exact location of the considered leak is marked using a black star. 


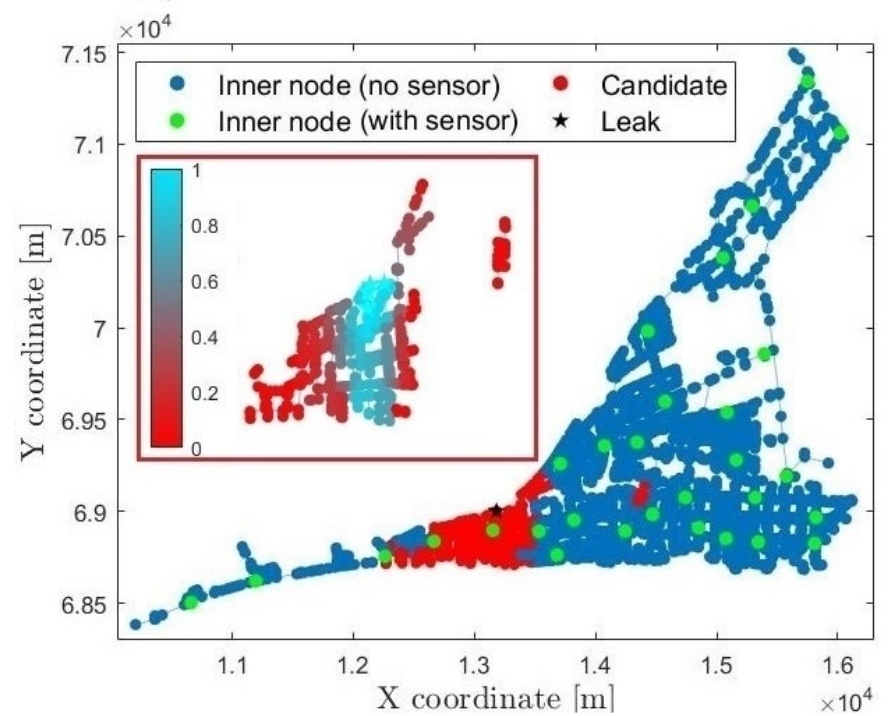

(a)

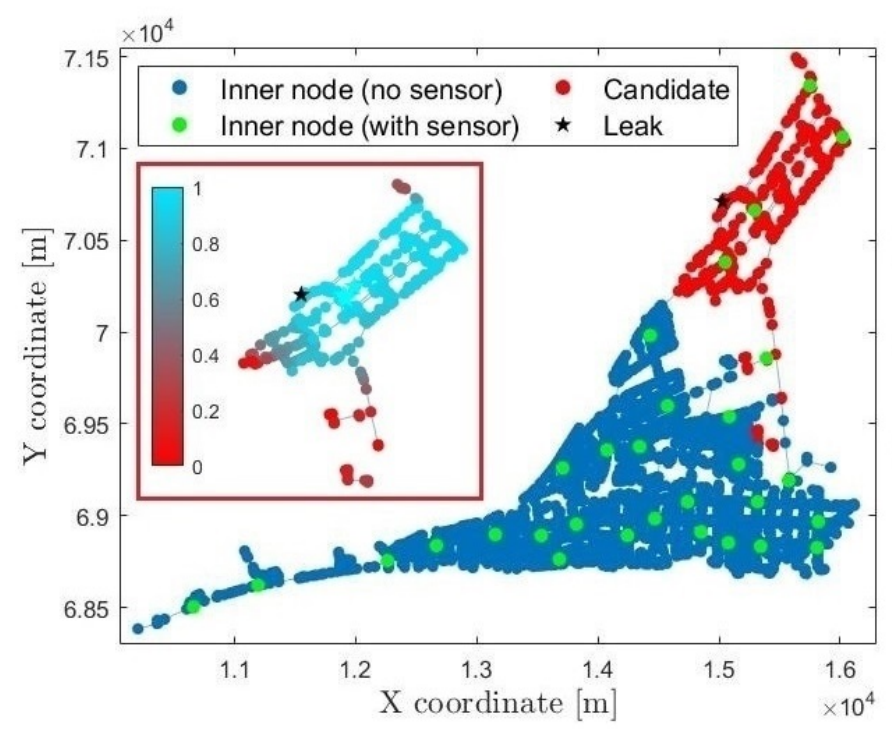

(c)

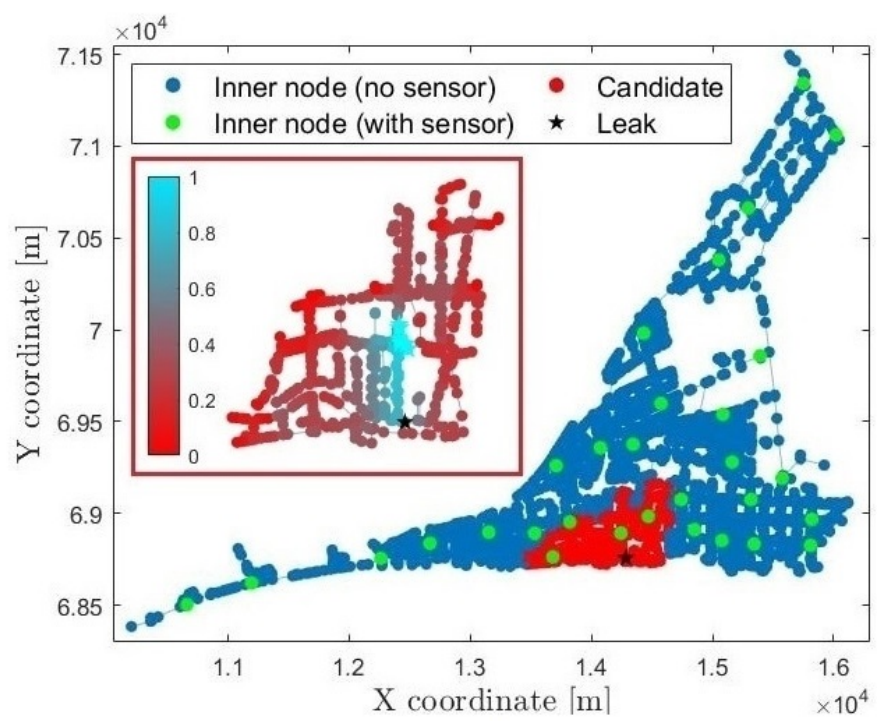

(b)

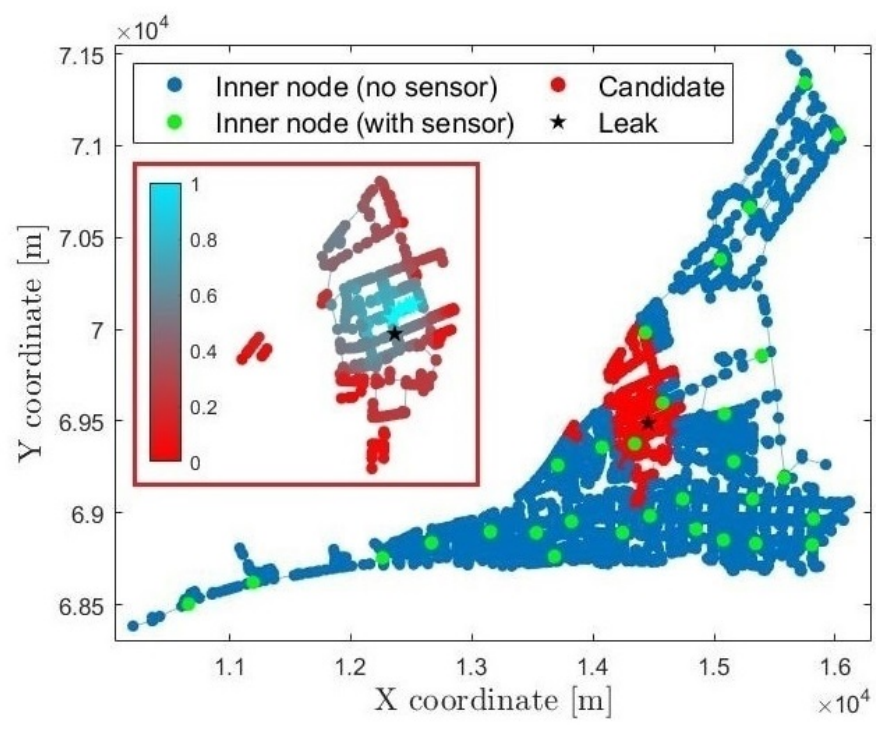

(d)

Fig. 3. Graphic representation of the application of the methodology over four leak events, scattered across the network.

On the other hand, a local perspective of the set of candidates is considered. The colour of a certain node encodes its relative value of distance in $\mathbf{r}$ with respect to the other candidates (the distance values of the candidates are normalized in the range $[0,1]$, and a colour bar is added to indicate the colour/value relation). Finally, the 10 best valued candidates are marked using cyan stars, in order to highlight them from the rest (the black star indicating the exact leak position is substituted by a cyan one in Fig. 3a because the leak is perfectly located).

Besides, the previously mentioned time integration approach is exploited. Therefore, the complete available time series of hydraulic measurements from the sensors is used, applying the methodology each time instant and adding the computed distance vector $\mathbf{r}$ to the sum of the previous ones. This operation reduces the appearance of outliers and the effect of the noise at the measurements, as well as it provides a set of candidates which is more independent of the boundary conditions of a certain time instant.

The provided information by the graphic representations in Fig. 3 is complemented with Table II, which compares several metrics for different number of sensors. The $\tau$ parameter in (9) is settled to 1000 for these tests.

TABLE II

PERFORMANCE RESULTS FOR DIFFERENT NUMBER OF SENSORS.

\begin{tabular}{|c||c||c||c|}
\hline $\boldsymbol{N}^{\boldsymbol{o}}$ sensors & $15(0.3 \%)$ & $30(0.6 \%)$ & $45(0.9 \%)$ \\
\hline Accuracy $(\%)$ & 87.878 & 96.969 & 100.000 \\
Candidates/network ratio & 0.136 & 0.135 & 0.123 \\
Distance to leak $(\mathrm{m})$ & 469.437 & 246.042 & 184.571 \\
\hline
\end{tabular}


These key performance indicators are computed by averaging the achieved values of these metrics for a set of leaks (of $2.5 \mathrm{l} / \mathrm{s}$ ) that are scattered across the network, as illustrated by Fig. 4. They are described in the following:

1) $N^{o}$ sensors: represents the number of installed sensors, that in the three presented cases include 15, 30 and 45 devices, implying a sensorization density of $0.3 \%$, $0.6 \%$ and $0.9 \%$ respectively.

2) Accuracy: it indicates the percentage of leaks that are correctly included in the set of candidates.

3) Candidates/network ratio: it is the quotient of the number of nodes in the set of candidates divided by the number of nodes of the complete network. It measures the "area" that the candidates occupy in the network.

4) Distance to leak: it represents the mean Euclidean distance from the leak to the 10 best candidates.

Considering both the graphic results from Fig. 3 and the ones provided by Table II, several aspects can be discussed:

- The methodology achieves a great accuracy regarding the inclusion of the leaky node into the candidates set.

- The localization area is greatly reduced, concretely to a $12-13 \%$ of the complete network. Despite this value can be higher for zones with a lower sensorization density (see Fig. 3c), adequately sensorized regions achieve an even more reduced localization area.

- The localization process tends to place the best candidates near to the closest sensor to the leak, due to the reduced sensorization density (see Fig. 3b). This is confirmed by the decrease in the distance-to-leak metric when the number of sensors augments. However, it is important to remark that the exact leak location is marked with blue dots in all the cases at Fig. 3, thus achieving a sufficiently good localization result.

- Outliers may still appear (see Fig. 3a), but their distance value in $\mathbf{r}$ is very low and hence they can be discarded.

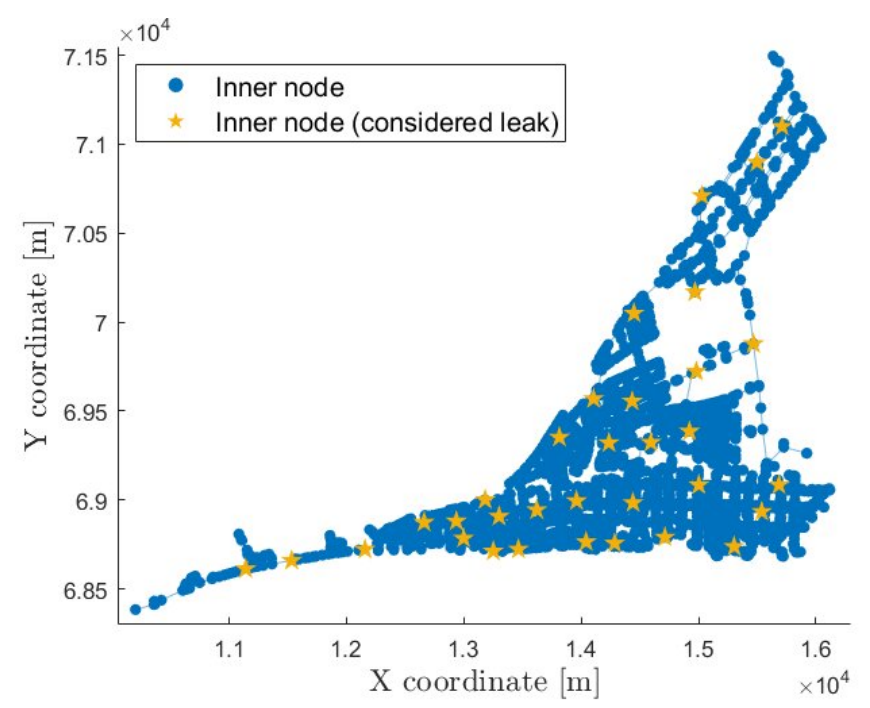

Fig. 4. Graph of the WDN that shows the locations of the leaks considered for the computation of Table II.

\section{CONCLUSIONS}

This article proposes a data-driven leak localization method based on the estimation of the state of the complete WDN from the hydraulic information gathered by a set of pressure sensors and the network topology.

The approach provides as result a set of candidates to be the leak origin, and additionally the leak exact location can be approximated by means of a computed metric.

A case study based on a real network is presented, showing the capability of the method to successfully locate the leak inside the set of candidates while greatly reducing the localization area (to approximately a $12-13 \%$ of the network), with a minimal amount of sensors in comparison with the total number of nodes (less than the 1\%) and the network structural information.

Therefore, the required investment for the installation of a sufficient number of sensors is justified by the savings in leaked water, workforce and the investment to develop and maintain a well-calibrated model of the network.

\section{REFERENCES}

[1] R. Puust, Z. Kapelan, D.A. Savić, and T. Koppel, A review of methods for leakage management in pipe networks, Urban Water J., vol. 7, no. 1, pp. 25-45, Feb. 2010.

[2] X. Leflaive, Water outlook to 2050: The OECD calls for early and strategic action, in Global Water Forum, May 2012.

[3] Q. Xu, R. Liu, Q. Chen, and R. Li, Review on water leakage control in distribution networks and the associated environmental benefits, J. Environ. Sci., vol. 26, no. 5, pp. 955-961, May 2014.

[4] M.W. LeChevallier, R.W. Gullick, M.R. Karim, M. Friedman, and J.E. Funk, The potential for health risks from intrusion of contaminants into the distribution system from pressure transients, J. Water Health, vol. 1, no. 1, pp. 3-14, Apr. 2003.

[5] T.K. Chan, C.S. Chin, and X. Zhong, Review of Current Technologies and Proposed Intelligent Methodologies for Water Distributed Network Leakage Detection, IEEE Access, vol. 6, pp. 78846-78867, Dec. 2018.

[6] R. Pérez, G. Sanz, V. Puig, J. Quevedo, M.À. Cugueró-Escofet, F. Nejjari, J. Meseguer, G. Cembrano, J.M. Mirats-Tur, and R. Sarrate, Leak Localization in Water Networks: A Model-Based Methodology Using Pressure Sensors Applied to a Real Network in Barcelona [Applications of Control], IEEE Control Syst. Mag., vol. 34, no. 4, pp. 24-36, Aug. 2014.

[7] S. Sophocleous, D.A. Savić, and Z. Kapelan, Leak Localization in a Real Water Distribution Network Based on Search-Space Reduction, J. Water Resour. Plan. and Manag., vol. 145, no. 7, pp. 04019024, July 2019.

[8] J. Blesa, and R. Pérez, Modelling uncertainty for leak localization in Water Networks, IFAC-PapersOnLine, vol. 51, no. 24, pp. 730-735, Oct. 2018.

[9] L. Ferrandez-Gamot, P. Busson, J. Blesa, S. Tornil-Sin, V. Puig, E. Duviella, and A. Soldevila, Leak Localization in Water Distribution Networks using Pressure Residuals and Classifiers, IFACPapersOnLine, vol. 48, no. 21, pp. 220-225, Oct. 2015.

[10] Q. Han, R. Eguchi, S. Mehrotra, and N. Venkatasubramanian, Enabling State Estimation for Fault Identification in Water Distribution Systems Under Large Disasters, in Proc. IEEE 37th Symp. on Reliable Distr. Syst. (SRDS), 2018, pp. 161-170.

[11] A. Soldevila, J. Blesa, T.N. Jensen, S. Tornil-Sin, R.M. FernándezCantí, and V. Puig, Leak localization method for water-distribution networks using a data-driven model and dempster-shafer reasoning, IEEE Trans. Contr. Syst. Technol., pp. 1-12, Apr. 2020.

[12] A. Soldevila, J. Blesa, R.M. Fernández-Cantí, S. Tornil-Sin, and V. Puig, Data-Driven Approach for Leak Localization in Water Distribution Networks Using Pressure Sensors and Spatial Interpolation, Water, vol. 11, no. 7, June. 2019.

[13] L.A. Rossman, The EPANET Programmer's Toolkit for Analysis of Water Distribution Systems, presented at the 29th Annu. Water Resour. Plan. and Manag. Conf., Tempe, AZ, June 6-9, 1999, 1-10. 